\title{
COLLOQUE INTERNATIONAL « PRATIQUES, TECHNiques ET THÉORIES DANS L'HISTOIRE DE LA GÉNÉTIQUE "
}

Nous vivons aujourd'hui dans « L'ère de la génétique ", suivant la formule de Marcel Blanc, la génétique est donc la science omniprésente de notre époque et, comme « ... on ne connaît pas complètement une science tant qu'on n'en sait pas l'histoire » (A. Comte), il nous a paru nécessaire d'instruire un public sur l'histoire d'une discipline scientifique qui le concerne soit directement (généticiens, médecins...), soit indirectement (historiens de la biologie, philosophes, sociologues, enseignants...).

Ce colloque d'histoire de la génétique a été nécessairement limité dans son contenu. Nous avons dû faire un choix dans les thèmes qui seront traités par des spécialistes, mais ces thèmes couvrent une période assez large de l'histoire de la génétique et permettent, de par leur diversité, de nous donner une image exacte de cette histoire pluridisciplinaire, et de dévoiler, par la même occasion, les préoccupations et le travail des historiens de la biologie.

Les différents thèmes de ce colloque seront traités dans l'ordre suivant : C. Sinding, La formation du « concept de gène » au XIX ${ }^{e}$ siècle ; B. Kimmelman, Agricultural Science and Mendelian Theory: Institutional Dynamics in America Genetics 1900-1915 ; J.-L. Fischer, Rôle des horticulteurs et agronomes français dans les débuts de la génétique (1910-1915) ; R. Olby, The Role of British Agriculture and Horticulture in the Establishment of Experimental Genetics; G.E. Allen, Race Horses and Draft Horses : Eugenics and Agriculture in the United States, 1900-1940 ; N. Roll-Hansen, Crossing the Pure Lines : From Johannsen to Nilsson-Ehle ; P.J. Weindling, Eugenics and German Geneticist ; M.H. Mandrillon, Eugénisme et hygiénisme en U.R.S.S., 1920-1940 ; M.B. Adams, Théories et pratiques dans le développement de la génétique des populations de l'U.R.S.S., 1920-1940 ; J. Gayon, Le formel et l'empirique dans l'histoire de la génétique des populations ; B. Fantini, Embryologie, gènes et acides nucléiques en 1930-1950 ; R.-M. Ponsot, Génétique et développement : liens établis par C.H. Waddington entre embryologie et génétique, 1939-1960 ; C. Galperin, Génétique et microbiologie : les problèmes de la lysogénie, 1925-1950 ; J. Sapp, Organisation, Cybernetics and the Problem of Cytoplasmic Inheritance ; R. Burian, French Contributions to the Research Tools of Molecular Genetics, 1945-1960 ; M. Morange, Histoire du concept de gène régulateur ; W. Schneider, The Role of Human Blood Groups in the History of Genetics ; et ce colloque s'achève par une synthèse sur la génétique actuelle : Ph. Kourilsky, Regards sur la génétique contemporaine.

Ce colloque va se tenir au Palais de la Découverte du 19 au 22 mai 1987. Il est organisé avec l'aide et le patronage du C.N.R.S., de l'U.N.E.S.C.O., de la Direction de la coopération scientifique et technique du Ministère des relations étrangères et de l'I.N.S.E.R.M. Le responsable de l'organisation est la Fondation "Pour la Science ", Centre international de synthèse, et la direction scientifique est assurée par J.-L. Fischer (C.N.R.S.).

La correspondance doit être adressée au Centre international de synthèse, 12, rue Colbert, 75002 Paris (tél. (1) 429750 68). 